\title{
EDUCACIÓN
}

\section{Metodologías y métodos para la creación de materiales didácticos para la enseñanza de lenguas extranjeras con el uso de las nuevas tecnologías}

\author{
Luís Eduardo Wexell Machado', Riciele Reis de Urbieta ${ }^{1}$
}

\section{Resumen}

Objetivo: Esta investigación tiene como objetivo presentar parte del proyecto de investigación en desarrollo: "Materiales didácticos para le enseñanza de lenguas extranjeras: creación de materiales impresos y digitales abiertos (REAs)", adjudicado a la Universidad Autónoma de Asunción y auspiciado por el Consejo Nacional de Ciencia y Tecnología (CONACYT) en lo que se refiere al planteamiento del uso de metodologías y métodos de enseñanza y aprendizaje de lenguas extranjeras con el uso de las nuevas tecnologías para un aprendizaje integrado. El proyecto ha sido elaborado a partir de la necesidad de profesores de portugués lengua extranjera de trabajar con materiales didácticos adaptados al contexto paraguayo, de costo accesible y de buena calidad. También trata de incorporar la percepción de los estudiantes paraguayos sobre su proceso de aprendizaje y del uso de materiales didácticos, bien como el uso de las nuevas tecnologías para el aprendizaje de lenguas extranjeras. La propuesta contempla el uso de metodologías activas: aprendizaje tangencial, aprendizaje basado en proyecto y aula invertida; del aprendizaje integrado (Seamless Learning), de la interculturalidad y del posmétodo como articuladores de la construcción de los materiales didácticos en un diseño educacional unificador. Los dos objetivos básicos que se quiere atingir con el proyecto son: 1) crear materiales didácticos significativos, de cualidad y de bajo costo para la enseñanza de lenguas extranjeras, en modalidad híbrida; 2). brindar unidad pedagógica a los materiales didácticos contemplando la diversidad de abordajes consideradas en el proyecto por medio de un diseño educacional para la enseñanza de lenguas en clases presenciales y a la distancia.

Material y Método: La metodología ha sido elaborada con base en un abordaje

\footnotetext{
1. Universidad Autónoma de Asunción, Paraguay.

Proyecto auspiciado por el Consejo Nacional de Ciencia y Tecnología de Paraguay - CONACYT. Un panorama general de este proyecto ha sido presentado en formato poster en la Sociedad Científica del Paraguay en Asunción, en agosto de 2017.

Una exposición oral del proyecto enfocando los aspectos del relevamiento de datos y la percepción de los estudiantes ha sido presentada en la Conferencia Internacional de Estudios del Lenguaje - Cielin 2017, octubre 2017, Universidad de Brasilia, Brasil.

Una exposición oral del proyecto enfocando la Interculturalidad en los Materiales Didácticos ha sido presentada en el IV Congresso Nacional de Estudos Linguísticos - IV CONEL, que se realizó los días 16 y 17 de noviembre de 2017, Universidad Federal de Santa Catarina, Brasil.

E-mail: lewmachado@gmail.com

DOI: 10.26885/rcei.foro.2017.93
} 
cualitativo por medio de una investigación-acción continuada a lo largo de dos años (2016/17) en que se realizó, de forma exploratoria, una encuesta con 118 estudiantes para evaluar su percepción con relación a los procesos de enseñanza y aprendizaje y el uso de los materiales didácticos y de las nuevas tecnologías en clase y extra-clase, además de una investigación narrativa con tres ex-estudiantes de portugués lengua extranjeras que siguen su formación académica en sus respectivas áreas, en Brasil en cursos de posgrado. La investigación narrativa ha sido realizada por medio de diversos encuentros, con uso de entrevistas gravadas y transcriptas en formato digital. El total de las entrevistas suman más de seis horas de grabación y han sido hechas de forma abierta. Además de la percepción de los estudiantes, se hizo un estudio de los cuadros de referencia para la enseñanza de lenguas extranjeras, más específicamente el Marco Común Europeo de Referencia para las Lenguas - MCERL, el American Council on the Teaching of Foreign Languages - ACTL y los Parámetros Curriculares Nacionales de Brasil- PCNs. También se ha hecho un análisis de los materiales didácticos para la enseñanza de portugués lengua extranjera que circulan en Paraguay.

Resultados: Los resultados parciales están materializados en seis secuencias didácticas, que comprenden dos niveles que conforman el ciclo básico de enseñanza, Portugués I y Portugués II y han sido elaborado con base en la licencia Creative Commons, en formato de Recursos Educacionales Abiertos (REAs), para atender a dos semestres lectivos y que podrán ser utilizadas en actividades preparadas para las clases presenciales y/o extra-clase con el uso de las nuevas tecnologías. Los materiales parten de la propuesta de una enseñanza de lengua extranjera de forma integrada: formal y no formal, presencial y a distancia, con materiales impresos y digitales, por medio de dispositivos fijos y móviles, de forma ubicua, y permiten extender el tiempo de aprendizaje y aumentar el nivel de personalización de la enseñanza. Los resultados parciales muestran que los materiales dinamizan las clases presenciales, fomentan la colaboración y la participación activa de los estudiantes y promueven el aprendizaje de la lengua en usos relacionados a situaciones reales y de acuerdo a las necesidades de los estudiantes. Las actividades extra-clase todavía necesitan mejor ajuste ya que un elemento primordial para su buen funcionamiento reside en factores relacionados con la motivación intrínseca y con la auto-gestión de su proprio aprendizaje por parte de los estudiantes. Cuestiones relacionadas con motivación, principalmente de naturaleza intrínseca, autonomía y agencia de los estudiantes deberán ser contempladas en la conclusión del proyecto con vista a mejorar los procesos de interacción en línea y la auto gestión del aprendizaje por parte de los estudiantes.

Palabras clave: portugués lengua extranjera, materiales didácticos, enseñanza integrada, metodologías activas, interculturalidad. 


\section{Referencias}

Chan, T. W. (2015). Removing Seams by Linking and Blurring How We Came Up with Seamless Learning. In M. Wong, L. H., Milrad \& M. Specht (Ed.), Seamless Learning in the Age of Mobile Connectivity (1a ed., pp. v-xv). London: Springer.

Kramsch, C. (1995). The cultural component of language teaching. Language, culture and Curriculum, 8(2).

Kumaravadivelu, B. (1994). The postmethod condition: (E)merging strategies for second/foreign language teaching. TESOL Quarterly, 28(1), 27-48.

Mendes, Edleise. (2011). Por uma formação intercultural de professores e alunos de PLE. In: E. Mendes (Ed.), Diálogos interculturais: ensino e formação em português língua estrangeira. Campinas - SP: Pontes Editores. 